\title{
HIGHLIGHTS
}

\section{Permafrost thawing and carbon metabolism}

\section{ce \\ microbial \\ ecology and carbon cycling in thawing permafrost}

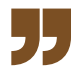

As a result of the rise in global temperatures, permafrost thaws and the sequestered organic material becomes accessible to microorganisms for degradation. As microbial processes contribute to the global flux of greenhouse gases, a better understanding of the long-term effects of permafrost thaw and microbial-mediated carbon cycling is needed so that these effects can be accounted for in predictive climate-change models. Three studies now provide insights into the microbial lineages involved in carbon processing in thawing permafrost, the role of viruses in soil carbon cycling, and the pattern, extent and scientific implications of sampling bias in environmental field research across the terrestrial Arctic.

In the first study, Woodcroft, Singleton et al. used a genome-centric metagenomic analysis of soil microbial communities from Stordalen Mire, Sweden, to examine how specific lineages transform organic matter during permafrost thaw. They collected 214 samples from three sites across a thaw gradient (an intact palsa, a partially thawed bog and a fully thawed fen) at different depths, and recovered 1,529 metagenomes from a diverse range of bacterial and

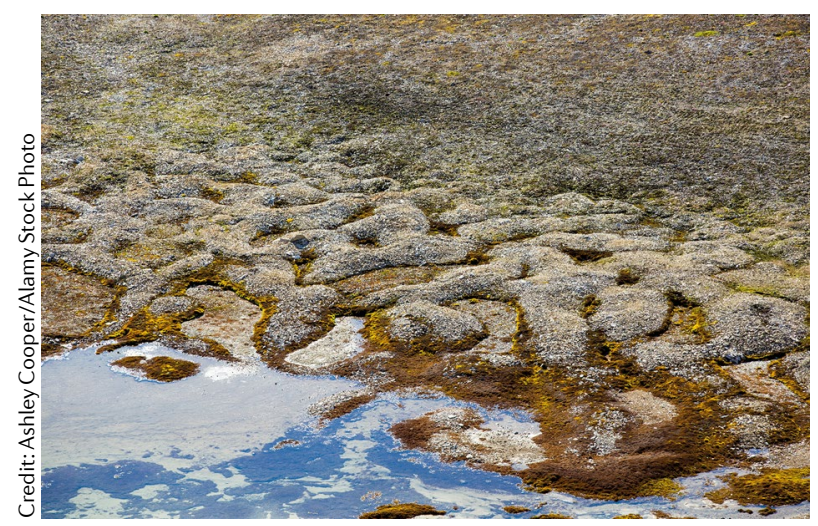

archaeal phyla. These phyla included Bacteria belonging to Actinobacteria and Acidobacteria, and Archaea from the Euryarchaeota, as well as many poorly characterized phyla. Community structures differed greatly between sites, and abundance varied at different depths in the soil column. Meta-omic analysis revealed key populations involved in the degradation of organic matter. Bacteria could degrade polysaccharides across all three thaw environments, including Acidobacteria, Proteobacteria, Ignavibacteriae and Bacteroidetes. Genes involved in lactate fermentation are encoded by actinobacterial and acidobacterial genomes in the palsa and bog, and a small proportion of the genomes at those sites encode proteins involved in ethanol and propionate fermentation. Most of the recovered archaeal genomes were identified as hydrogenotrophic methanogens, suggesting that they are the greatest contributor to the formation of methane $\left(\mathrm{CH}_{4}\right)$. The high abundance of methanotrophs in the bog suggests that they oxidize a substantial proportion of $\mathrm{CH}_{4}$, thus limiting emissions to the atmosphere.

In the second study, Emerson et al. investigated how viruses influence microbial ecology and carbon metabolism at the three sites at Stordalen Mire. They detected 1,907 viral populations in bulk soil metagenomes, more than half of which were also detected in metatranscriptomes; these data suggest that many of these viruses are active. $35 \%$ of the viral populations could be linked to hosts from 19 bacterial and archaeal phyla and included viruses of methanogens, methanotroph viruses and viruses of respiring and fermentative heterotrophs, indicating that they indirectly affect carbon cycling, possibly by infecting and eliminating microbial carbon cyclers. Lineage-specific virushost abundance ratios differed significantly between habitats, which suggests that permafrost thaw may affect virus-host infection dynamics. Further evidence indicating that viruses affect complex carbon degradation comes from the identification of predicted viral genes encoding glycoside hydrolases, which degrade plant-derived polymers into monosaccharides and oligosaccharides that can be used in microbial carbon degradation pathways to generate $\mathrm{CH}_{4}$ and $\mathrm{CO}_{2}$. Finally, viral abundance was shown to predict climate-relevant carbon chemistry measurements (such as methane dynamics).

In a third study, Metcalfe et al. report that a substantial proportion of the Arctic remains undersampled. They emphasize the need to increase both the quality and quantity of sampling, and to incorporate existing literature from poorly cited areas, to better represent the effects of climate change on arctic environments.

Together, these studies highlight that a deeper understanding of microbial ecology and carbon cycling in thawing permafrost will aid to accurately predict climate-induced changes.

Andrea Du Toit

ORIGINAL ARTICLES Woodcroft, B. J.,

Singleton, C. M. et al. Genome-centric view of carbon processing in thawing permafrost. Nature https://doi.org/10.1038/s41586-018-0338-1 (2018) | Emerson, J. B. et al. Host-linked soil viral ecology along a permafrost thaw gradient. Nat. Microbiology https://doi.org/10.1038/s41564 018-0190-y (2018) | Metcalfe, D. B. et al. Patchy field sampling biases understanding of climate change impacts across the Arctic. Nat. Ecol. Evol. https://doi.org/10.1038/s41559-018-0612-5 (2018) 